\title{
Clinicopathological Review of Resectable Gastric Cancer at a Tertiary Level Teaching Hospital of Nepal
}

\author{
Prasan Kansakar, Pradeep Vaidya \\ Department of Gl and General Surgery, Maharajgunj Medical Campus, Tribhuvan University Teaching Hospital, \\ Institute of Medicine, Kathmandu, Nepal
}

\section{Corresponding author:}

Prasan Kansakar, MS, MCh

Department of GI and General Surgery, Maharajgunj Medical Campus, Tribhuvan University Teaching Hospital, Institute of Medicine, Kathmandu, Nepal

Email:pbskansakar@hotmail.com

\begin{abstract}
Introduction

According to available data, gastric carcinoma is the most common malignancy arising from the gastrointestinal tract in Nepal. Our objective was to analyze the pathological features of gastric carcinoma in patients undergoing curative resection.
\end{abstract}

\section{Methods}

Retrospective analysis of histopathological reports of patients who underwent curative surgery for gastric cancer was performed at Department of Surgery, Tribhuvan University Teaching Hospital for a period of two years. Demographic characteristics, symptoms, site of tumor, type of surgery performed, macroscopic and microscopic characteristics including number of harvested lymph nodes, number of positive lymph nodes and tumor stage were analyzed.

\section{Results}

A total of 36 patients underwent curative resection. Mean age of the patients was 61.6 years with range of 21-82 years. Male:female ratio was 7:4. Pain abdomen was the predominant presenting symptom. Vomiting, hematemesis and malena were the other frequent symptoms. Endoscopy Borrmann type II tumor was seen in 27 (75\%) of patients. Most patients underwent subtotal gastrectomy. In 25 patients, tumor was located in antrum. Lymphovascular and perineural invasion was seen in 21 and 20 patients respectively. Sixteen patients had poorly differentiated tumors. All but two patients had microscopic negative distal margin whereas proximal margin was negative in all patients. A mean of 15 lymph nodes were harvested. Twenty three patients had node positive disease. Pathological staging revealed Stage IA in 3 patients, Stage IB in in 5 patients, stage IIA in 4 patients stage IIB in 6 patients, stage IIIA in 6 patients, stage IIIB in 8 patients and IIIC in 4 patients.

\section{Conclusion}

Gastric carcinoma was common in seventh and eighth decade of life with male preponderance. Most of the patients presented with locally advanced gastric cancer. Tumors located in antrum requiring subtotal gastrectomy was the predominant finding.

Keywords: Gastric cancer, pathological report, resectable tumor 